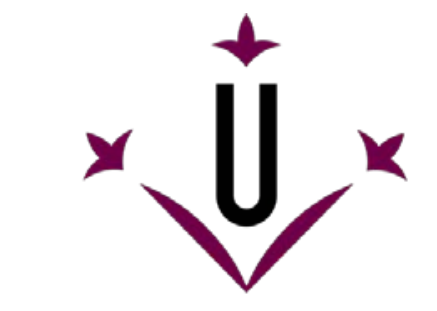

Universitat de Lleida

Document downloaded from:

http://hdl.handle.net/10459.1/65041

The final publication is available at:

https://doi.org/10.1109/TASC.2016.2591825

Copyright

(c) IEEE, 2016 


\title{
H-Formulation FEM Modelling of the Current Distribution in 2G HTS Tapes and its Experimental Validation Using Hall Probe Mapping
}

\author{
G. G. Sotelo ${ }^{1,2}$, M. Carrera ${ }^{3}$, X. Granados. ${ }^{2}$ \\ ${ }^{1}$ Electrical Engineering Department, Fluminense Federal University (UFF), Brazil \\ ${ }^{2}$ Institut de Cièncias de Materials de Barcelona (ICMAB CSIC), Barcelona, Spain \\ ${ }^{3}$ Dep. Medi Ambient i Ciències del Sòl, Universitat de Lleida (UdL), Lleida, Spain \\ *Email: gsotelo@id.uff.br; mcarrera@macs.udl.cat; granados@icmab.es
}

\begin{abstract}
:
One of the most widespread mathematical formulations applied to simulate the electromagnetic phenomena of coated conductor in the recent literature is the $\mathrm{H}$ one. However, the only validation of the model has been indirect by using measurements taken from the applications, as measurements of the energy losses in ac fields, forces developed in levitation systems or any other parameter related to a specific application. Direct validation of the calculation requires the observation of the local out of plane magnetic field over the surface of the sample and this is only accessible under magnetooptical observations and, in a larger scale and better dynamic range, by the Hall scanning microscopy. We propose here the experimental validation of the $\mathrm{H}$ formulation by comparing the simulated results with measurements made by a Hall probe mapping in a second generation (2G) tape sample for several DC transported currents at $77 \mathrm{~K}$. The paper presents a methodology to simulate the $2 \mathrm{G}$ tape by using only measured data obtained from a sample and its normalized $J(B)$ experimental curves. Some boundary conditions that allow a faster convergence of the problem are investigated. Simulated results of the $2 \mathrm{G}$ tape modelled considering only the $1 \mu \mathrm{m}$ HTS layer were compared with other that represent the most important layers of the coated conductor structure in the calculations. The simulated and measured results present a good agreement, proving that this model can calculate precisely the magnetic field and, hence, the current distribution in HTS samples.
\end{abstract}

\section{Keywords:}

FEM, H-formulation, Superconducting modelling, 2G tape, Hall magnetometry. 


\section{Introduction}

The recent coated conductors quality improvements allowed to reach a new level of technology development in superconducting large scale applications [1-2]. In order to design devices using superconducting tapes, it is necessary to use computational tools to simulate those materials considering the nonlinearity intrinsically associated to them. The modeling of high temperature superconductors (HTS) was vastly investigated in the literature, and there are several formulations applied to solve the electromagnetic problem. The formulations more frequently found in the literature applied to solve the problem by the finite element method (FEM) are: the $\mathrm{H}$ [3-5], the $\mathrm{A}-\mathrm{V}$ (or $A-\phi$ ) [6-8], the $\mathrm{T}-\Omega$ [9-11] and the $\mathrm{E}[12]$ formulations. All those formulations were used to calculate the current distribution inside the superconductor for bulks and tapes. For the case of the coated conductors, one of the models applied frequently in the recent literature is based on the magnetic field strength, also named as H-formulation. One reason for that is the simplicity to implement it in FEM commercial programs, like COMSOL.

Considering the $\mathrm{H}$-formulation, the previous works dedicated to the validation of the model only contrasted the calculated results with measurements provided by a mean value of the current distribution, which represents an indirect comparison. Generally, the results are presented with good agreement [4, 13-16] for the energy dissipated (power losses) in a superconducting tape transporting an AC current. Also, the $\mathrm{H}$ formulation by FEM simulations was successfully compared with levitation force measurements [17]. Besides, the simulated results for those models were compared with analytical equations $[4,18]$.

The current distribution inside a superconducting tape is directly associated with the external magnetic field produced by it, which can be obtained by mapping its surface using a Hall probe. Using an inversion method based on the Biot-Savart's law, the current distribution in a HTS tape was obtained [19-20].

In this context, the present work has the following contributions: 
i) To validate the calculated current distribution inside a $2 \mathrm{G}$ tape obtained by the $\mathrm{H}$ formulation comparing it with Hall probe mapping measurements;

ii) To simulate the $2 \mathrm{G}$ tape only using experimental parameters easily obtained from a sample and the manufacturer data;

iii)To investigate the influence of relevant parameters in the simulated results, such as consideration of the layers in the modelling, and comparison between different boundary conditions.

\section{The modeling of $2 \mathrm{G}$ tapes}

\subsection{The H-formulation}

The $\mathrm{H}$-formulation has the advantage of solving directly the differential equations for the magnetic field. It has been extensively presented in the literature to calculate the current distribution in superconductors [3-5, 13-16,18]. By the Faraday's law, for a 2D symmetry presented in Fig. 1, where $\mathrm{L}$ is considered infinity long and only the cross section is simulated, it is possible to write the following two partial differential equations (PDEs):

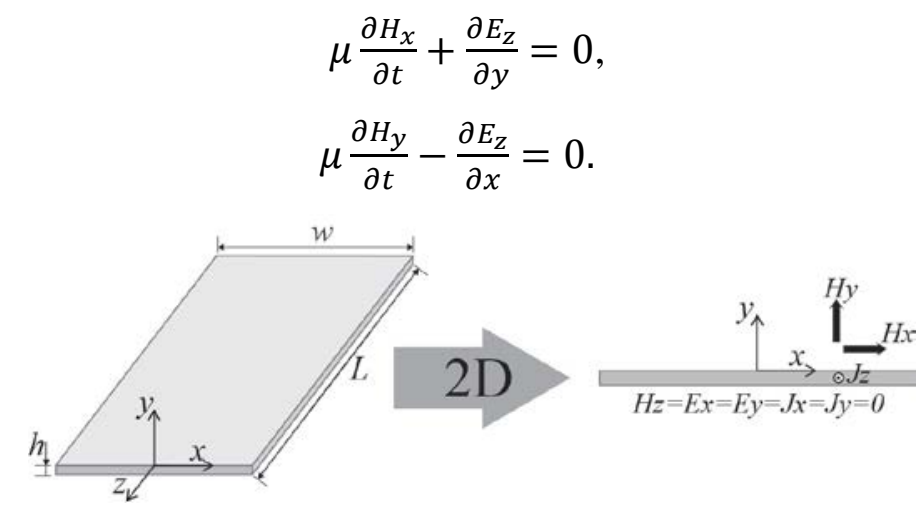

Fig. 1: The simulated symmetry in $2 \mathrm{D}$ for the $2 \mathrm{G}$ tape.

By the Ampere's law (neglecting the displacement current) for the symmetry presented in Fig. 1, and the power law, it is possible to obtain a constitutive equation to represent the flux creep and flux flow in the superconducting region, as follow:

$$
E_{z}=\frac{E_{c}}{J_{c}}\left|\frac{J_{z}}{J_{c}}\right|^{n-1} \cdot\left(\frac{\partial H_{y}}{\partial x}-\frac{\partial H_{x}}{\partial y}\right)
$$

where $\mathrm{E}_{\mathrm{c}}$ will be adopted here as $1 \mu \mathrm{V} / \mathrm{cm}$, and the critical current density $J_{c}$ and $n$ are functions that can be obtained by experimental results or by analytical expression with adjusted parameters. 
To solve the problem, equations (1a), (1b) and (2) can be introduced in a software with capacity to resolve generic PDE. In the case of the present work, the problem was solved by the PDE module in COMSOL 4.4, in terms of the magnetic field components ( $H_{x}$ and $H_{y}$ ). For the air region, the first term in the right side product of equation (2) was replaced by $1 \Omega \cdot \mathrm{m}$ constant, and it must be nonzero in order to avoid a singularity in the system of equations [12]. It is an issue introduced to force a low resistivity air value and obtain the problem convergence.

\subsection{Boundary conditions and multilayer modeling}

The type of element used, nodal (also named Lagrangian) or edge (also known as rotational), will influence in how the boundary condition should be imposed to the problem and also in how fast the solution is calculated [21]. The application of the edge elements in the $\mathrm{H}$-formulation intrinsically guarantees that the $\nabla \cdot \mathrm{B}=0$ condition is satisfied [4], since this condition is present in the solution of each element. Furthermore, it was presented previously that both element types can present the similar results [22]. Several arguments were presented in [4-5] showing the advantages of the edge elements, since it presents a better distribution of the matrix and a faster convergence.

In this work the edge elements will be used. Three cases will be simulated and compared, in order to investigate the effects on the calculations of the boundary conditions for a reduced or a large domain, and the representation of only the HTS region or all the layers in the $2 \mathrm{G}$ tape, as follow:

i) to model the 2G tape only considering a $1 \mu \mathrm{m}$ HTS layer and an external reduced air domain, applying the magnetic field in the borders calculated by the Biot-Savart's law as Dirichilet boundary condition, named as integralequation-FEM coupling;

ii) to model the $2 \mathrm{G}$ tape considering the most important layers (HTS, substrate, silver and copper) in its structure and the same boundary conditions of case (i);

iii) to model the $2 \mathrm{G}$ tape representing the same layers of case (ii) and applying the Dirichilet boundary condition of $H_{x}=H_{y}=0$ in all external lines very far from the superconductor. 
The boundary condition of cases (i) and (ii) was originally proposed in [18]. It reduces the number of DOF by working with a narrow external air domain, in other words, by having the borders very close to the superconductor. The magnetic field is separated in two components, to be applied in the external border lines:

$$
\begin{aligned}
& H_{x}(x, y, t)=H_{x}^{e x t}(x, y, t)+H_{x}^{H T S}(x, y, t), \\
& H_{y}(x, y, t)=H_{y}^{e x t}(x, y, t)+H_{y}^{H T S}(x, y, t) .
\end{aligned}
$$

The variables $H_{x}^{e x t}$ and $H_{y}^{e x t}$ correspond to the external magnetic field in the $x$ and $y$ directions respectively. In the present work, no external field will be applied, and so $H_{x}^{\text {ext }}=H_{y}^{e x t}=0$. The variables $H_{x}^{H T S}$ and $H_{y}^{H T S}$ correspond to the magnetic field produced by the currents flowing through the superconductor.

For cases (i) and (ii), an intrinsic property of the edge elements is explored, since they have a null component along the perpendicular direction to their edges [4-5]. It simplifies the implementation of the boundary conditions, since equations (3a) and (3b) are respectively applied in $x$ and $y$ border lines (see Fig. 2). By the Biot-Savart's law, the magnetic field produced by the $2 \mathrm{G}$ tape currents can be obtained as follow:

$$
\begin{aligned}
& H_{x}^{H T S}\left(x_{1}, y_{1}, t\right)=\int_{S}\left(\frac{J_{z}(x, y, t) \cdot\left(y_{1}-y\right)}{\left(x_{1}+x\right)^{2}+\left(y_{1}-y\right)^{2}}-\frac{J_{z}(x, y, t) \cdot\left(y_{1}-y\right)}{\left(x_{1}-x\right)^{2}+\left(y_{1}-y\right)^{2}}\right) d S, \\
& H_{y}^{H T S}\left(x_{1}, y_{1}, t\right)=\int_{S}\left(\frac{J_{z}(x, y, t) \cdot\left(x_{1}-x\right)}{\left(x_{1}-x\right)^{2}+\left(y_{1}-y\right)^{2}}-\frac{J_{z}(x, y, t) \cdot\left(x_{1}-x\right)}{\left(x_{1}+x\right)^{2}+\left(y_{1}-y\right)^{2}}\right) d S .
\end{aligned}
$$

The simulations presented in this paper are centered in a geometry that corresponds to a 2G tape of $12 \mathrm{~mm}$ wide and $110 \mu \mathrm{m}$ thickness, with the $x$ and $y$ external borders of 6 $\mathrm{mm}$ and $1 \mathrm{~mm}$ respectively, as shown in Fig. 2.

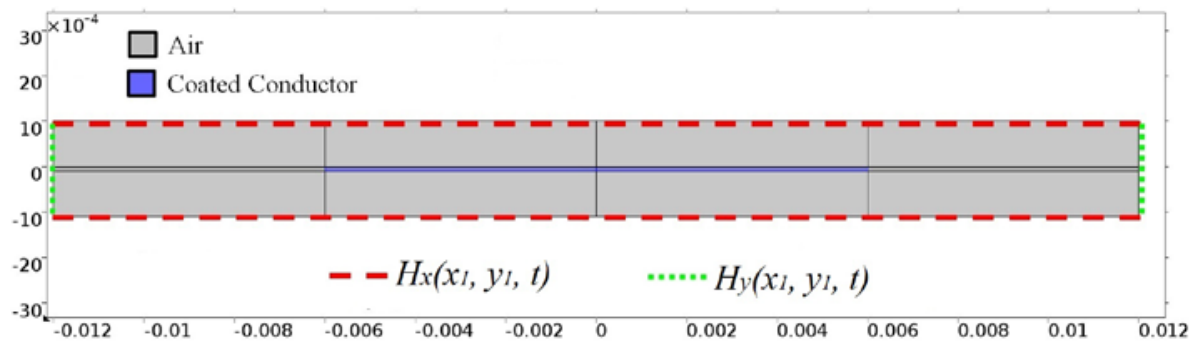

Fig. 2: The simulated 2G tape geometry and the Dirichilet boundary conditions integral-equation-FEM coupling applied to the problem. 
In case (iii), an external box for the air domain with $0.1 \times 0.1 \mathrm{~m}^{2}$ was introduced over the geometry of Fig. 2 that became centralized into it. The boundary condition of this case is to apply $\mathrm{H}_{\mathrm{x}}=\mathrm{H}_{\mathrm{y}}=0$ along the external box lines.

In order to calculate the current density distribution inside the tape, the value of the net current must be inserted in the formulation. In the case of the COMSOL software, it allows this constraint to be enforced by means of Lagrange multipliers, as described in [4], forcing the result of the net current to be equal to the current density integral over the HTS cross section area.

\subsection{Critical Current Dependence with the Field}

There are several ways to model the critical current distribution dependency with the magnetic field. The more general way is the consideration of an analytic expression for $J(B)$ with adjusted parameters [23], in order to fit the simulated and measured results. Table I present some of the analytical equations found in the literature to represent $J_{c}(B)$.

Table I: Analytical equations presented in the literature to represent the $J_{c}$ dependency with the external field.

Model for $\mathrm{J}_{\mathrm{c}}$ dependency and/or references Equation

Kim model [24]

$$
J_{c}(|\vec{B}|)=\frac{J_{c 0} \cdot B_{0}}{|\vec{B}|+B_{0}}
$$

Power-law [25,26]

$$
J_{c}(|\vec{B}|)=\frac{J_{c 0}}{|\vec{B}|^{\alpha}}
$$

Linear model [27]

$$
\begin{aligned}
& J_{c}(|\vec{B}|)=J_{c 0} \cdot\left(\frac{B_{0}-|\vec{B}|}{B_{0}}\right) \text {, if }|\vec{B}|<B_{0} \\
& J_{c}(|\vec{B}|)=0, \text { if }|\vec{B}| \geq B_{0}
\end{aligned}
$$

Exponential model [28]

$$
J_{c}(|\vec{B}|)=J_{c 0} \cdot \exp \left(-\frac{|\vec{B}|}{B_{0}}\right)
$$


Adapted Kim and Power-law [13, 29-30]

$$
J_{c}(|\vec{B}|)=\frac{J_{c 0}}{\left(1+\frac{\sqrt{k^{2} B_{x}^{2}+B_{y}^{2}}}{B_{0}}\right)^{\alpha}}
$$

Adapted Kim and Power-law with elliptical anisotropy [31-32]

$$
\begin{gathered}
J_{c, k}(|\vec{B}|, \theta)=\frac{J_{c 0, k}}{\left(1+\frac{|\vec{B}| f_{k}(\theta)}{B_{0, k}}\right)^{\alpha_{k}}} \text { and } \\
f_{k}(\theta)=\sqrt{v_{k}^{2} \cos ^{2}\left(\theta-\delta_{k}\right)+u_{k}^{2} \sin ^{2}\left(\theta-\delta_{k}\right)}
\end{gathered}
$$

Instead of choosing some of the models presented in Table I, we are proposing to use directly the normalized $J_{c}(B)$ curve expression for a 2G tape. This curve can be measured for a given sample.

The $I_{c}$ angular dependence is typically described by the manufactures, by giving two sets of curves, corresponding to $B / / c$ and $B / / a b$. In the actual state of the art, the successful introduction of pining centers induces a different angular behavior, but the pattern is well known as its temperature dependence. Both, the temperature dependence with $B / / C$ and $B / / a b$ and the angular pattern are currently available at the main produces.

Measurements of $J_{c}(\theta, B, T)$, are somewhat difficult. They are made typically in terms of $I_{c}$. One of the points of difficulty is originated by the self-screening effects of the tape, which induce a hysteretic behavior. In order to measure the intrinsic value, characterization is made in small micrometric wide bridges to approach it, neglecting so the screening effects and currents distribution that occurs in the wider samples. Main manufactures provide this kind of measurements at different temperatures and magnetic induction.

For $2 \mathrm{G}$ advanced pinning tapes operating at $77 \mathrm{~K}$ with magnetic flux density under $100 \mathrm{mT}$, the observed anisotropy effect is not so severe, allowing an approximating of the problem without considering the angular dependence of the current density. In our case, the $I_{c}(B)$ data were obtained from the manufacturer [33] for an applied DC magnetic field. The critical current was divided by the coated conductor area and 
normalized for the zero field value. The data is introduced in the program in a lookup table that interpolates the intermediate values, defined here as $J_{c} B$. This interpolation is applied to the HTS domain as a variable $J_{c}=J_{c 0} \times J_{c} B\left(\left|H \mu_{0}\right|\right)$, where $J_{c 0}$ is the critical current density at zero field and $\left|H \mu_{0}\right|$ is the local magnetic field magnitude. The variable $J_{c}$ is applied in each element to obtain the solution of the problem.

There are two reasons for modelling the problem in this way: it is not necessary to adjust any parameters present in the analytical equation and it was observed a faster convergence than using one of the expressions presented in Table I.

The data were introduced as a table for linear interpolation as shown in Fig. 3. Only the $J_{c}(B)$ component parallel to the c axis was implemented in our model. Obviously the modeling of angular dependence could better represent the physics of the problem, but it was neglected here for simplicity.

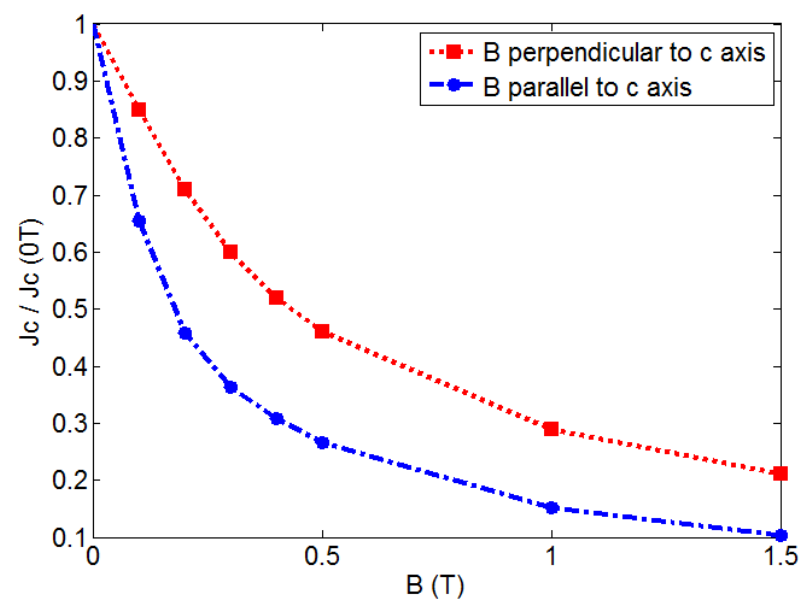

Fig. 3: Normalized critical current density data obtained from measured results [33] for a $2 \mathrm{G}$ tape similar to the studied in this work.

\section{Results}

\subsection{Current Density Distribution in a 2G Tape}

All the parameters used in the simulations were based in a commercial 2G tape. The sample tested was produced in 2015 by SuperOx and its dimensions are: 12 mm width, $110 \mu \mathrm{m}$ thickness and $170 \mathrm{~mm}$ long. More information about it and other parameters used for the simulation are presented in Table II.

Table II: Structural and electrical parameters of the $2 \mathrm{G}$ sample used in this work. 


\begin{tabular}{lc}
\hline Parameter & Value \\
\hline Wide & $12 \mathrm{~mm}$ \\
Total thickness & $110 \mu \mathrm{m}$ \\
HTS thickness & $1 \mu \mathrm{m}$ \\
Silver thickness & $2 \mu \mathrm{m}$ \\
Copper thickness (in both sides) & $20 \mu \mathrm{m}$ \\
Substrate (Hastelloy C-276) thickness & $60 \mu \mathrm{m}$ \\
Silver resistivity [34] & $2.7 \mathrm{n} \Omega \cdot \mathrm{m}$ \\
Copper resistivity [34] & $1.97 \mathrm{n} \Omega \cdot \mathrm{m}$ \\
Hastelloy C-276 resistivity [35] & $1.25 \mu \Omega \cdot \mathrm{m}$ \\
Critical current measured, self-field & $447 \mathrm{~A}$ \\
@ 77K (1 $\mu$ V/cm) & \\
Power-law parameter n (by fitting \\
with the measurements) \\
$\begin{array}{l}\text { Working temperature } \\
\end{array}$
\end{tabular}

The 3 simulated cases introduced in section 2.2, are summarized in Table III. A coarse and a fine meshes were implemented to solve the problem. The main difference between those meshes is the number of divisions along the horizontal lines ( $x$ direction). The coarse and the fine meshes have, respectively, 30 and 50 divisions along the $12 \mathrm{~mm}$ of the tape width. In all the simulated cases the superconductor layer was modeled with 1 $\mu \mathrm{m}$ thickness and 3 divisions were made to have three layers for the superconductor thickness ( $y$ direction). All the cases described were simulated in a computer with a processor i7-4790 (4 cores with 3.6 GHz) and $16 \mathrm{Mb}$ of RAM.

Table III: Simulated cases comparing boundary conditions and modeled layers.

\begin{tabular}{cccc}
\hline Case & $\boldsymbol{i}$ & $\boldsymbol{i i}$ & $\boldsymbol{i \boldsymbol { i }}$ \\
\hline Several Layers & No & Yes & Yes
\end{tabular}




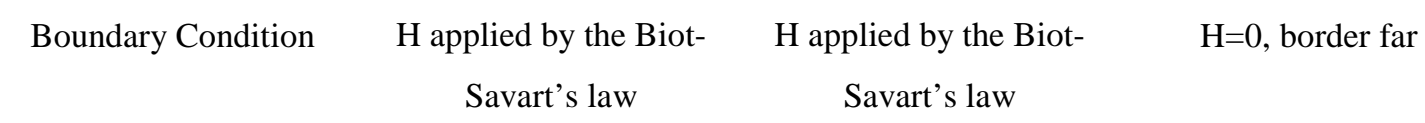

Mesh Coarse

\begin{tabular}{|c|c|c|c|}
\hline DOF & 1903 & 2794 & 4309 \\
\hline $\begin{array}{c}\text { Number of domain } \\
\text { elements }\end{array}$ & 920 & 1360 & 2412 \\
\hline $\begin{array}{c}\text { Number of boundary } \\
\text { elements }\end{array}$ & 275 & 490 & 512 \\
\hline Calculation time & $57 \mathrm{~min}$ & $2 \mathrm{~h}$ and $34 \mathrm{~min}$ & 1 day and $3.7 \mathrm{~h}$ \\
\hline \multicolumn{4}{|c|}{ Mesh Fine } \\
\hline DOF & 4253 & 6244 & 8811 \\
\hline $\begin{array}{l}\text { Number of domain } \\
\text { elements }\end{array}$ & 2070 & 3060 & 4846 \\
\hline $\begin{array}{c}\text { Number of boundary } \\
\text { elements }\end{array}$ & 475 & 890 & 914 \\
\hline Calculation time & 3h and $8 \mathrm{~min}$ & 1 day and $21 \mathrm{~h}$ & $\begin{array}{c}\text { > } 5 \text { days } \\
\text { (not concluded) }\end{array}$ \\
\hline
\end{tabular}

In all simulated cases, only first order edge elements were used. For cases (i) and (ii), the mesh was created only using quadrilateral mapped elements. For case (iii), the mesh in the areas presented in Fig. 2 is the same of the one made in case (ii), and additionally a free triangular mesh was created externally to this region.

In the simulations, the current was applied in the tape by a sequence of step functions, with an interval of $1 \mathrm{~s}$ to reach the next value and keeping the signal constant during 99 s, as presented in Fig. 4. The current was maintained constant during this time in order to allow the flux creep effects pass and to stabilize its distribution along the superconductor. 


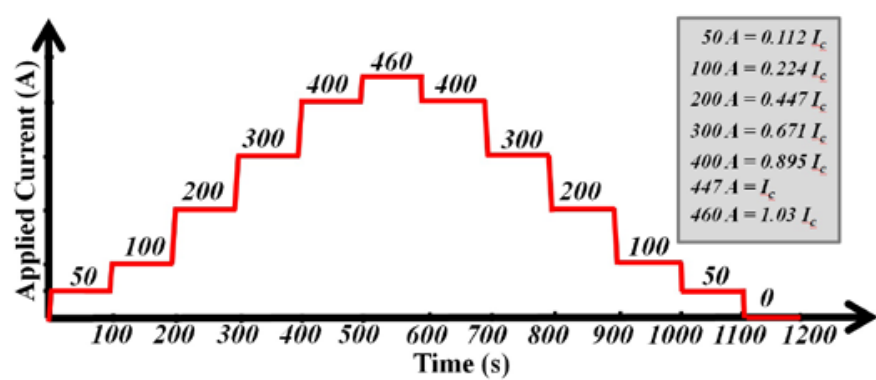

Fig. 4: Applied current profile in the superconducting tape during simulations and experiments.

A variable time step (BDF) was used in COMSOL without imposing limits. As there is not current variation during a long time, the simulated time step increases for these intervals, reducing the computational time.

Two cut lines were made inside the HTS $1 \mu \mathrm{m}$ layer: L1 in the center of the superconducting layer $(0.5 h)$ and L2 close to external border $(h / 6)$, where $h$ is the thickness according to Fig. 1. The current distribution inside the HTS layer along L1 and L2 are compared for the cases (i), (ii) and (iii). Fig. 5 presents the normalized current density calculated for the coarse mesh along L2 (Fig. 5 a) and L1 (Fig. 5 b), for the applied current profile of Fig. 4 during the rise. The results are presented for a time of $99 \mathrm{~s}$ after the previous step, when the current distribution is stabilized inside the superconductor. Fig. 6 presents the same results for the current decreased.

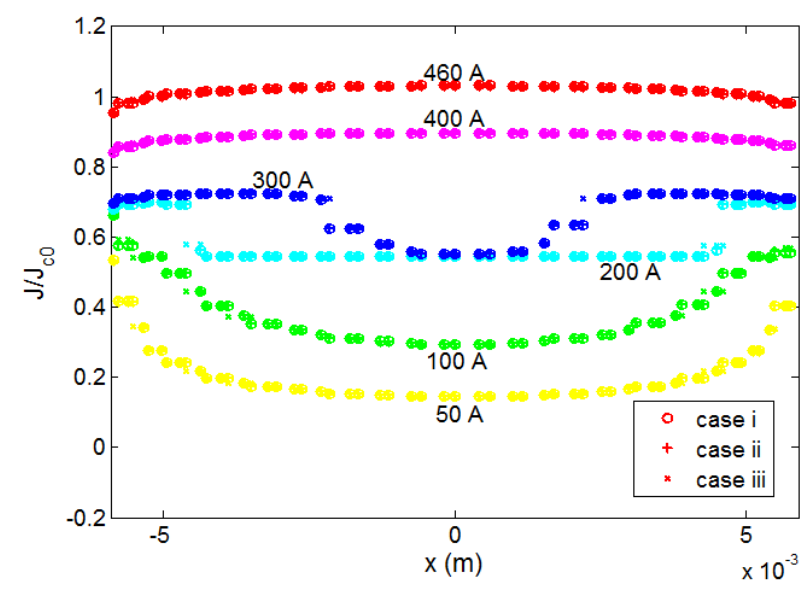

(a) 


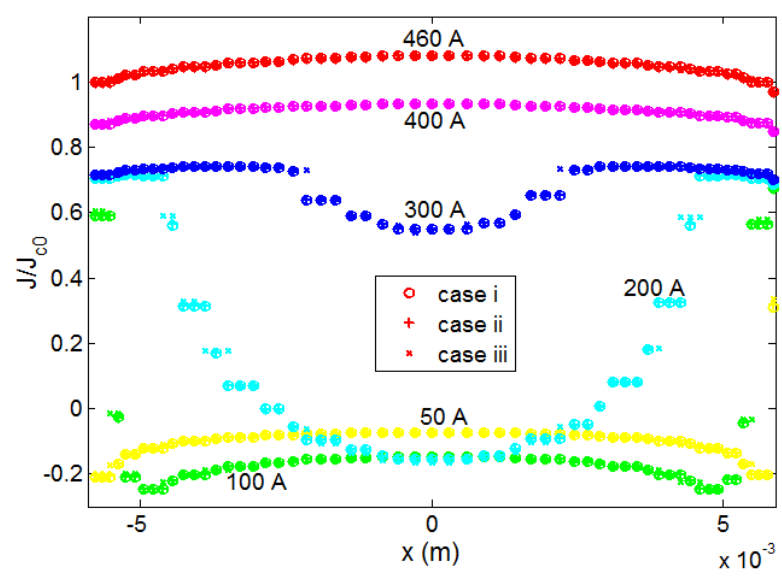

(b)

Fig. 5: Normalized current density along the superconducting layer when the applied current (Fig. 4) is increased: (a) line close to the HTS border (L2), (b) line at the center of the HTS layer (L1).

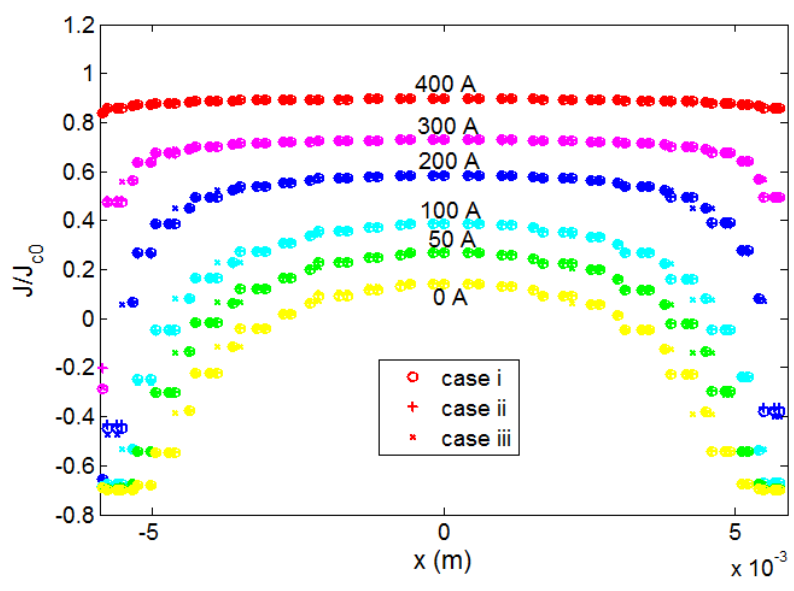

(a) 


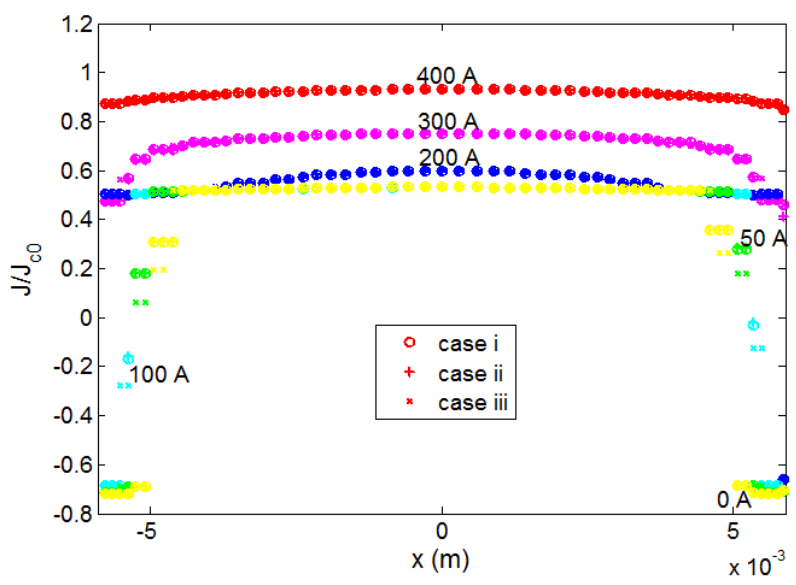

(b)

Fig. 6: Normalized current density along the superconducting layer when the applied current (Fig. 4) is decreased: (a) line close to the HTS border (L2), (b) line at the center of the HTS layer (L1).

It is important to note that the three simulated cases presented the same results for the current density distribution along all studied regions, except for minor numerical error in some points. It means that the consideration of placing the border close to the HTS and apply the magnetic field on it, implemented in cases (i) and (ii), worked well. Besides, there are not differences in the current distribution when the superconductor was modeled with only the superconductor layer (case $i$ ) or the other layers were considered (case ii). Actually, the difference in the current distribution between cases (i) and (ii) would appear if a value much higher than the critical current was simulated. In the results of Fig. 5, the current of 460 A (only 3\% over $I_{c}$ ) was not enough to make the difference evident. Fig. 7 presents the results of the normalized current distribution for L1 and L2 for 99 s after the time step, summarizing the results of Figs. 5 and 6. 


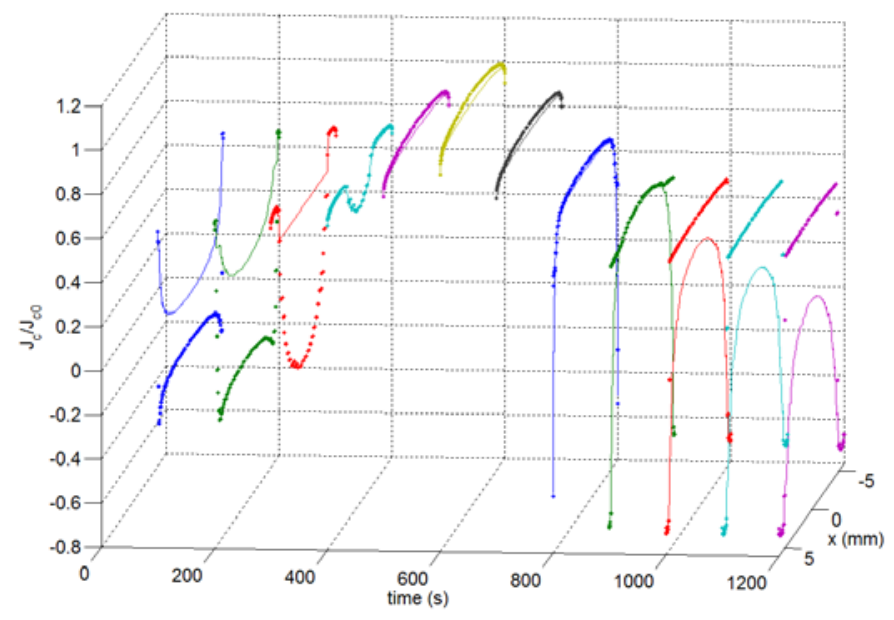

Fig. 7: Normalized current density for the current profile of Fig. 4. The continuous line and the dots represent L2 (border line) and L1 (central line), respectively.

Fig. 8 compares the simulated $B_{y}$ profiles for the coarse and fine meshes. The coarse mesh results show some unreal pointed peaks, which do not correspond to the measured profile. In the other hand, the fine mesh presents a profile shape in agreement to the experimental one, but the computation time increases as presented in Table III. From now on, all the simulated results will be presented for the case (ii) and the fine mesh.

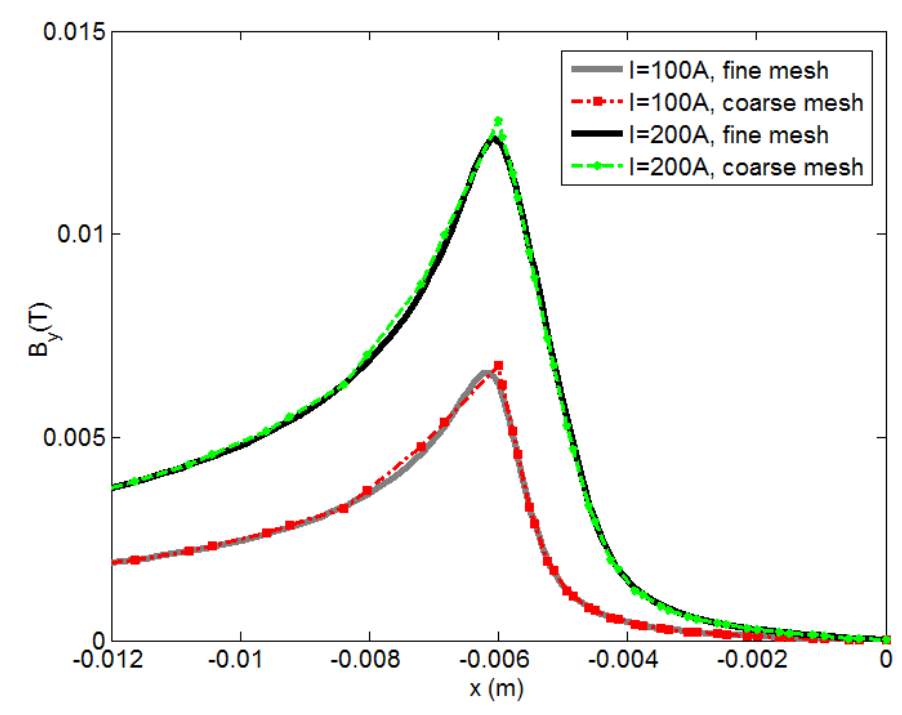

Fig. 8: Comparison of the calculated external magnetic induction results for the two simulated meshes for a distance of $450 \mu \mathrm{m}$ above the superconducting layer.

The formulation used in this work can take into account the flux creep effects observed in experiments with the superconducting material. The capacity of calculating precisely 
this phenomenon is a sine qua non condition for any model to be implemented in several 2G tape applications. After a step in the applied current (Fig. 4), the flux relaxation will make the current density reduce in magnitude and penetrates more in the tape. Since the flux creep has a logarithmic behavior, after some time the current density variation can be neglected. Fig. 9 presents the normalized current density for several instants along the cut lines L1 and L2. The arrows show the evolution of the current, where each line corresponds to $1 \mathrm{~s}$ frame, during $99 \mathrm{~s}$. The same result is presented in Fig. 10 for a 300 A current. It can be noted that the flux creep effect is stronger for 300 A than for 200 A case.

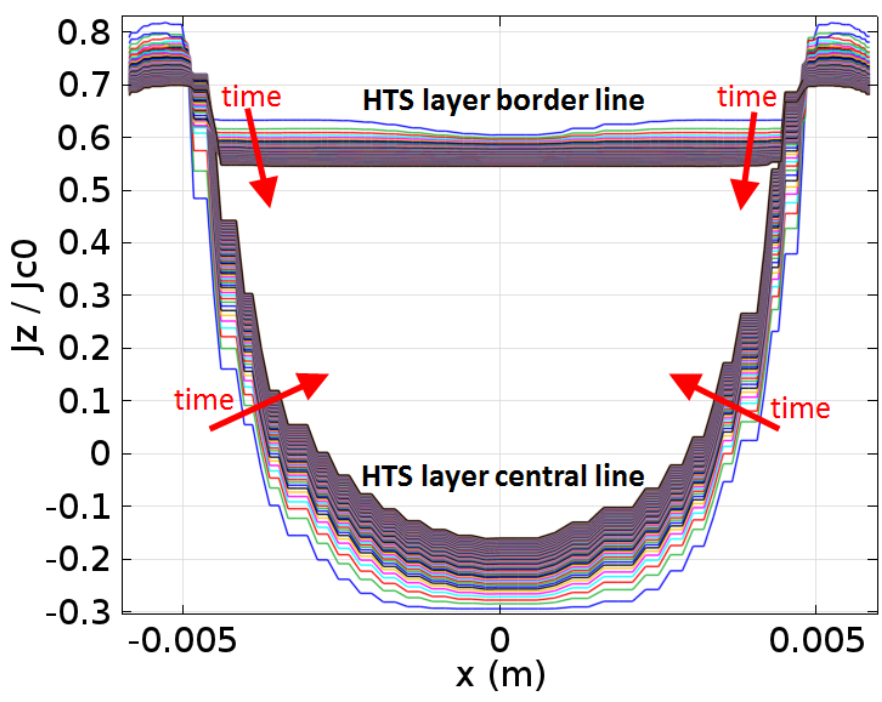

Fig. 9: Normalized current density for I = 200 A inside the superconducting layer along two crossing lines, central (L1) and border (L2), during 99 seconds.
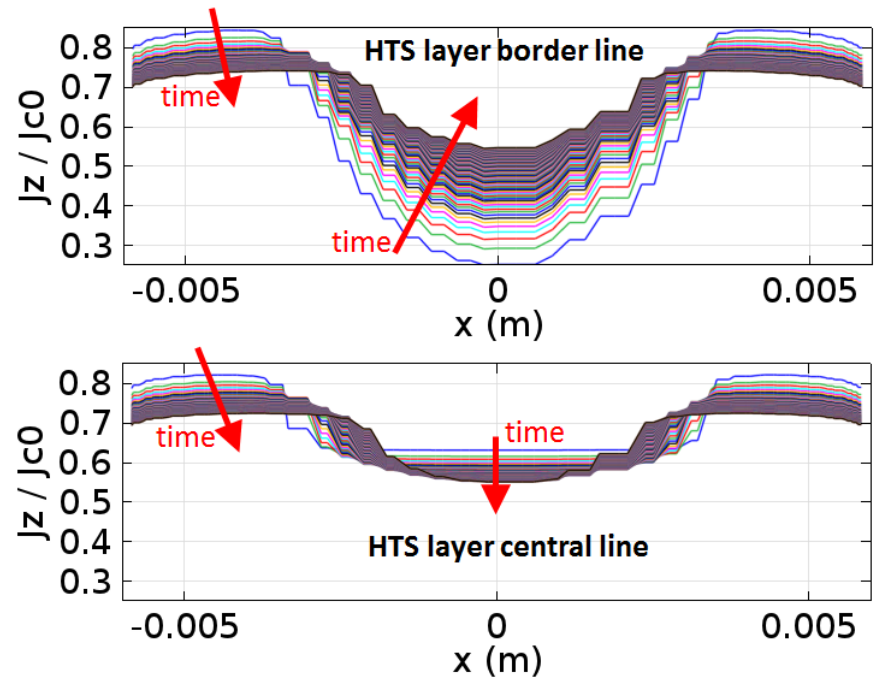
Fig. 10: Normalized current density for I = 300 A inside the superconducting layer along two crossing lines, central (L1) and border (L2), during 99 seconds.

Fig. 11 shows the temporal evolution of the external magnetic flux density in the $y$ direction for 200 A and 300 A applied currents during $99 \mathrm{~s}$, for a distance of $450 \mu \mathrm{m}$ above the superconducting layer. The arrows indicate the evolution of the magnetic induction along the time.

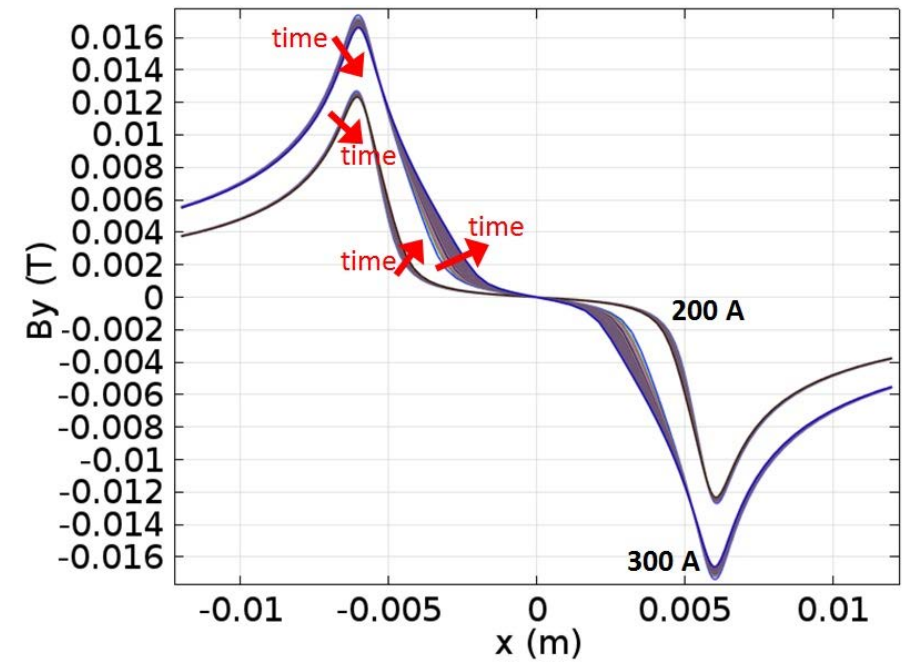

Fig. 11: Temporal evolution of the calculated magnetic induction profile, for $200 \mathrm{~A}$ and 300 A applied currents, and $450 \mu \mathrm{m}$ above the superconducting layer.

To investigate the effect in other currents, Fig. 12 presents the value of the magnetic induction in the position of the maximal value of the profile $(x=-6 \mathrm{~mm})$ for several different currents as a function of the time. Fig. 12 shows that the decay of the magnetic induction for $300 \mathrm{~A}$ is higher than for the others currents, indicating that the flux creep is more intense for currents in this value. This figure also presents that after $99 \mathrm{~s}$, the value of the magnetic induction is almost stabilized, and this time is enough to compare the simulated results with the measurements, which will be presented in the next section. 


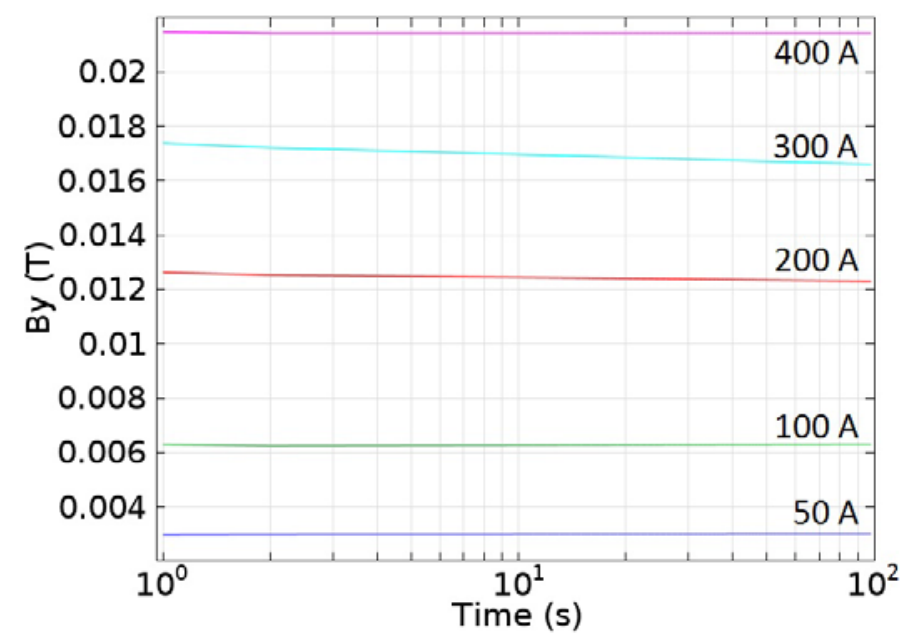

Fig. 12: Magnetic induction decay in the peak value $(x=-6 \mathrm{~mm})$ of the $B_{y}(x)$ profile after the current step.

\subsection{Model Validation}

This section presents the Hall scanning magnetometer [36-37] results on a 2G tape sample and compare them with the FEM simulations. A Hall probe sensor placed in a flexible strip was adjusted to gently touch the sample. In this condition, the active part in the sensor (active area of $300 \times 300 \mu \mathrm{m}^{2}$ ) has a distance of $400 \mu \mathrm{m}$ from the sample surface. And so, the distance from the HTS layer and the active part of the Hall sensor is approximately $450 \mu \mathrm{m}$. For this reason, the simulated results will be presented for $y=450 \mu \mathrm{m}$ (Fig. 1). To make the mapping of the magnetic induction $B_{y}(x, z)$, the Hall sensor was placed in a $x y z$ system. All the measurements were made with the tape and the sensor in a LN2 bath (77K). The Hall mapping system precision is $0.01 \%$ for full scale range of $0.5 \mathrm{~T}$.

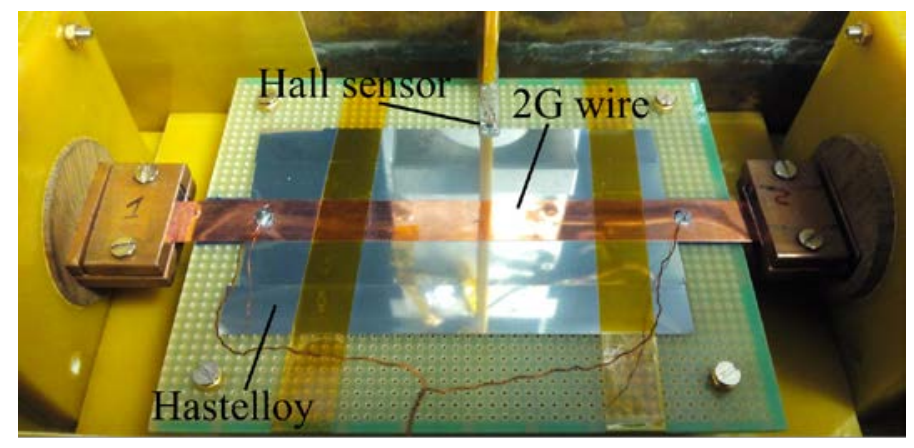

Fig. 13: Experimental rig designed to measure the external field produced by a transport current in a $2 \mathrm{G}$ tape sample. 
The magnetic induction scanning was made for the current profiles presented in Fig. 4. After each current was applied, an interval of time higher than $120 \mathrm{~s}$ was waited before the map was started. However, it is not a problem to compare the simulated and measured results, since the magnetic induction practically does not change after a longer time, as presented in Fig. 12, due to the logarithmic behavior of the flux creep.

To determine the critical current a nanovoltmeter was used to measure the voltage between two points separated $10 \mathrm{~cm}$ in the sample. The V(I) curve was measured in the sample as presented in Fig. 14, in order to obtain the critical current $\left(I_{c}\right)$ and the $n$ exponent of equation (2). The determined $I_{C}$ is $447 \mathrm{~A}(1 \mu \mathrm{V} / \mathrm{cm})$ and the $n$ exponent is 32, after fitting the measured data.

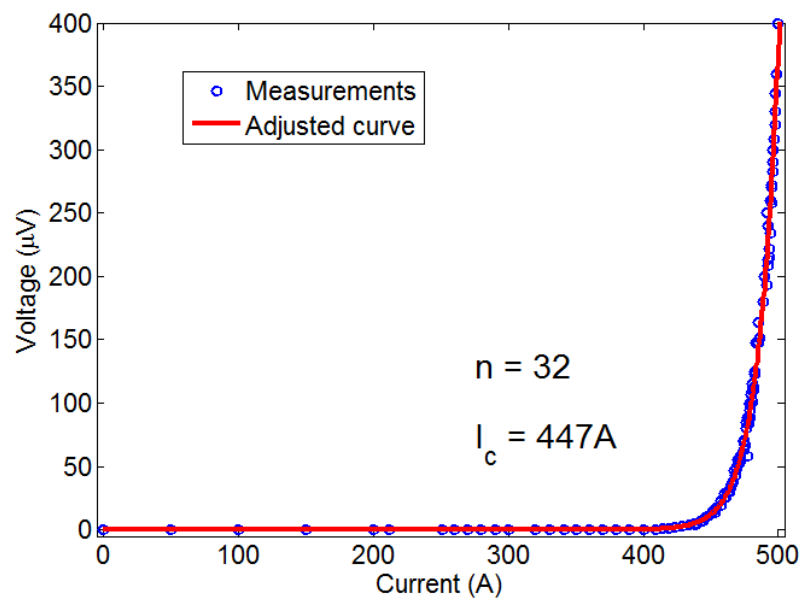

Fig. 14: V(I) measured and its fitted curves for the 2G tape sample.

Figs. 15, 16 and 17 present the comparison between the simulated and measured results for all the currents of Fig. 4. As can be observed the measured and simulated results are agreeing, indicating that the applied $\mathrm{H}$-formulation can reproduce well the phenomena. We can observe that the results of Fig. 15, for increasing currents up to $300 \mathrm{~A}$, present a better agreement than the results of Figs. 16 (currents closer to $I_{c}$ ) and 17 (decreasing currents). For the current rise (Fig. 15 and Fig. 16 a and b) the maximum observed discrepancy was about $10 \%$. For the current drop (Fig. 16 c and d and Fig. 17) the maximum discrepancy was about $30 \%$, however the model continues to predict correctly the physical phenomenon. It can be also observed that the measurements do not present the curves as perfectly symmetrical in relation to the $x=0 \mathrm{~m}$ position as the 
simulations. The observed asymmetry should be attributed to some small imperfections in the sample.

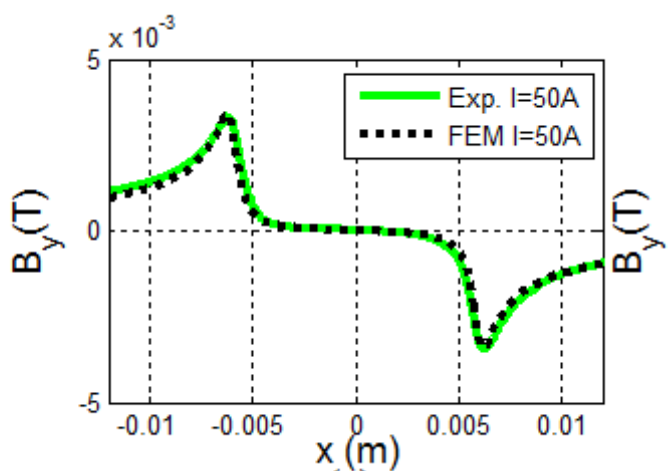

(a)

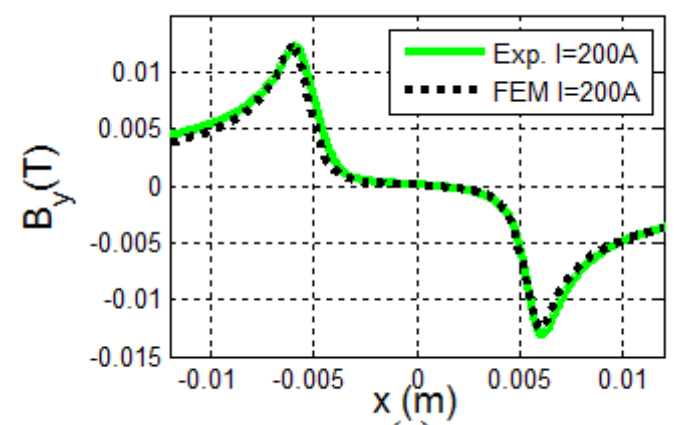

(c)

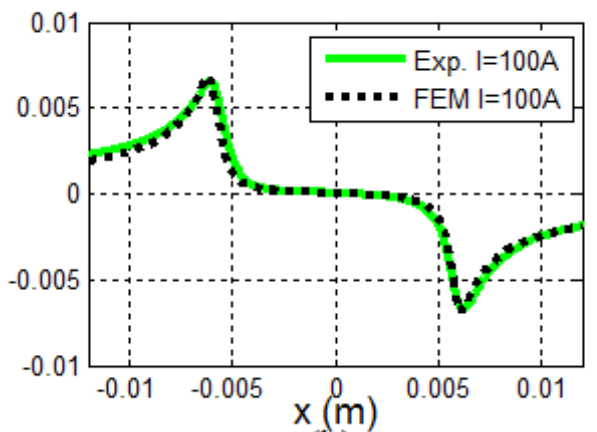

(b)

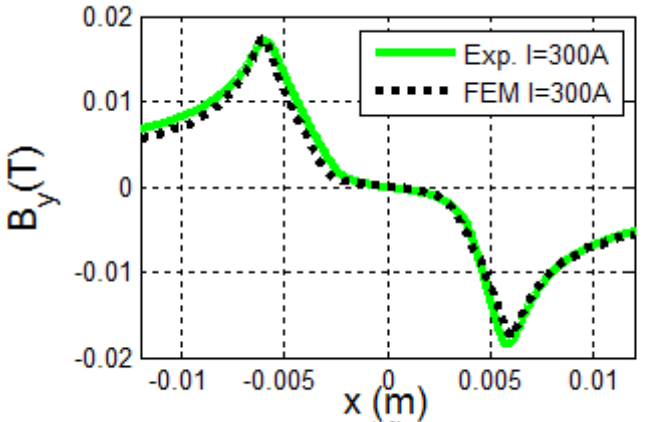

(d)

Fig. 15: Simulated and measured magnetic induction profiles for currents among $50 \mathrm{~A}$ and 300 A during its rise. 


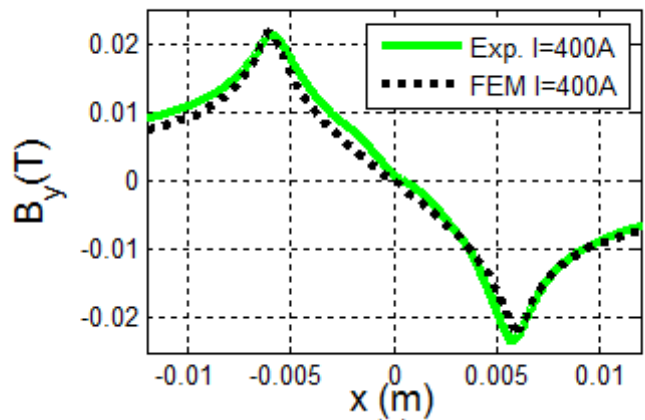

(a)

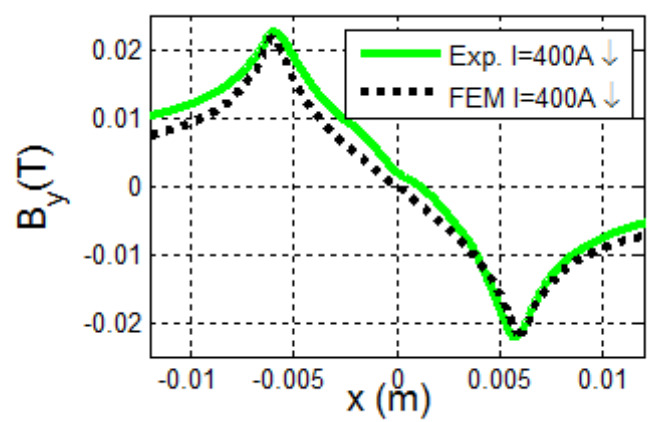

(c)

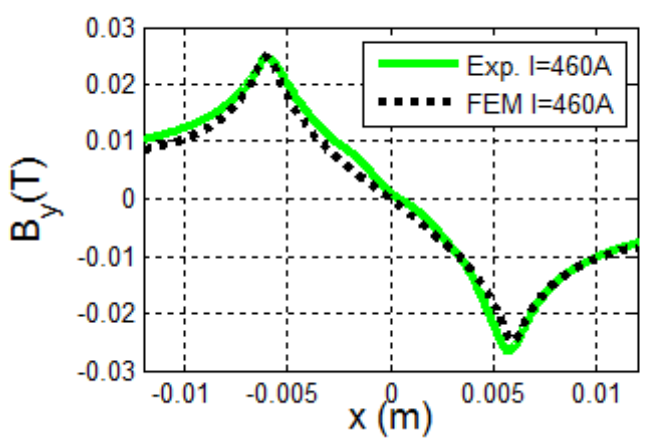

(b)

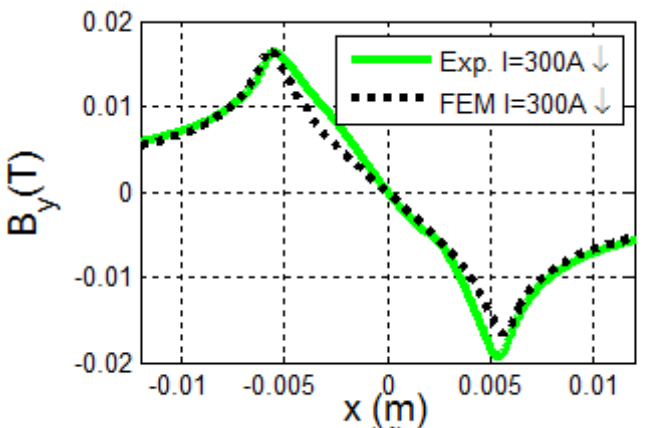

(d)

Fig. 16: Simulated and measured magnetic induction profiles for currents of: (a) $400 \mathrm{~A}$ (rise), (b) $460 \mathrm{~A}$ (maximal), (c) $400 \mathrm{~A}$ (drop) and (d) $300 \mathrm{~A}$ (drop).

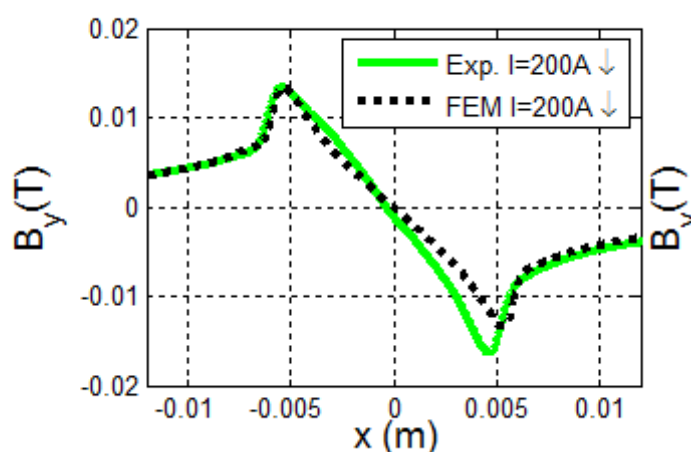

(a)

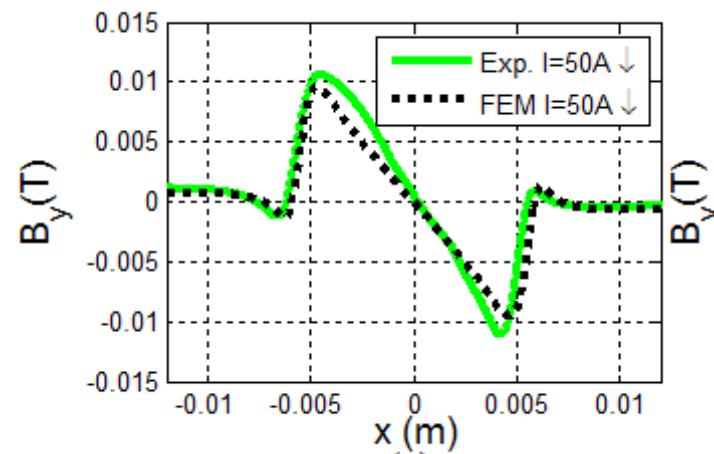

(c)

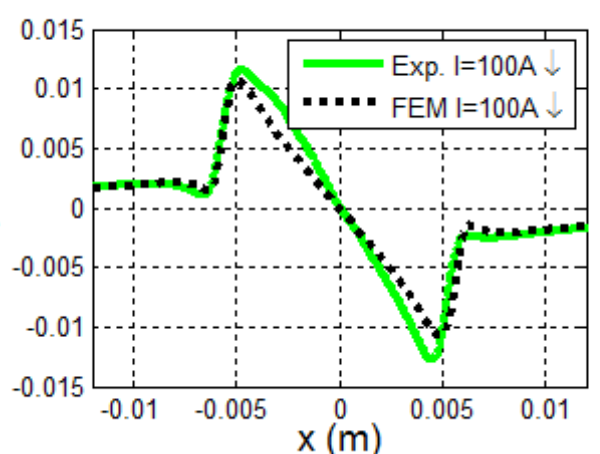

(b)

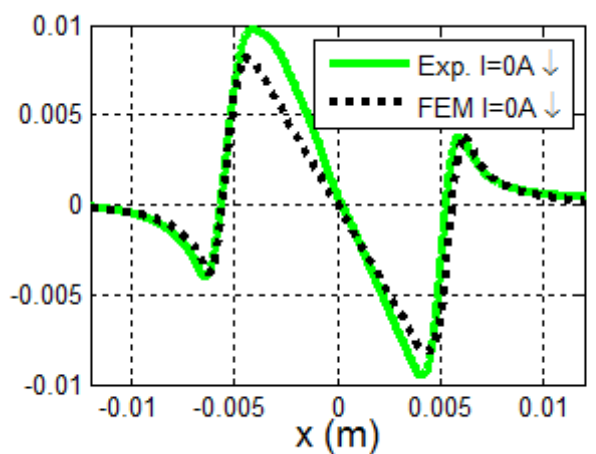

(d) 
Fig. 17: Simulated and measured magnetic induction profiles for currents among $200 \mathrm{~A}$ and 0 A (remnant field) during its drop.

The sample inhomogeneity can be estimated by a magnetic induction scanning map of an extension area. Figs. 18 and 19 show a $B_{y}$ (x,z) map for 400 A (during the current rise) and $0 \mathrm{~A}$ (remnant field), respectively. In both figures it is possible to observe that the magnetic field profile is not exactly the same for two different $\mathrm{z}$ positions. The maximum fluctuation observed in the measured peak value of the 400 A map (Fig. 18) was about $6 \%$. For the remnant state (Fig. 19) this oscillation along the measured peak values was about $16 \%$. It means that if an adjustment in the simulation parameters were made to reproduce a determined experimental result for one cross section along $\mathrm{z}$, the same adjust would not fit exactly to another line.

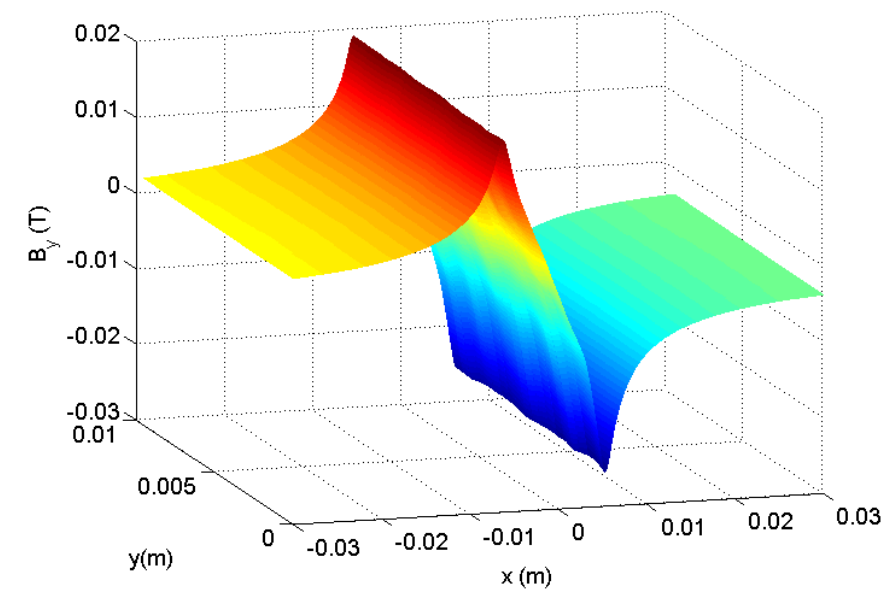

Fig. 18: Magnetic induction scanning for 400 A during the current rise.

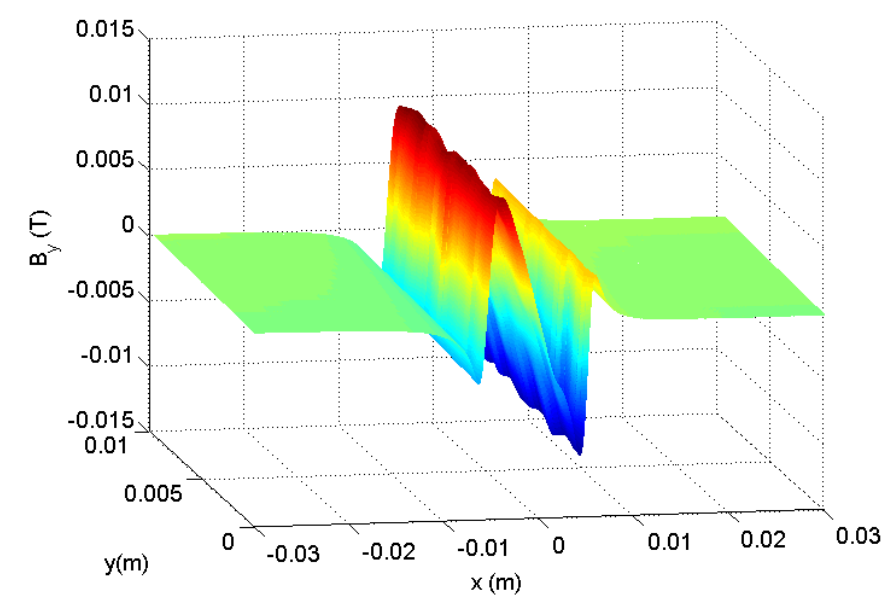


Fig. 19: Magnetic induction scanning for 0 A (remnant field) after drop the current.

\section{Conclusion}

This work presented a validation of the H-formulation applied to calculate the current distribution in a $2 \mathrm{G}$ tape. The simulations were made only using parameters obtained from the experimental results. Instead of using any analytical expression with adjusted parameters for the critical current dependency with the field, the normalized $J_{c}(B)$ data, obtained from the $I_{C}(B)$ curves supplied by the manufacturer, was applied directly in the computation code. The $J_{c}$ angular dependency in the 2G tape was neglected here.

The critical current $I_{c}$ and the $n$ exponent were obtained from the $\mathrm{I}(\mathrm{V})$ curve measured in a sample, and these parameters were introduced into the simulation code. By the applied methodology it is not necessary to determine any constant to adjust the simulated data in order to fit with the experimental results with the calculations.

Two boundary conditions were compared: a boundary far from the HTS with zero field condition and a boundary close to the HTS with an imposed field in the border determined by the Biot-Savart's law (integral equation-FEM coupling). The calculated current distribution inside the $2 \mathrm{G}$ tape was the same for both cases, but it was possible to reduce drastically the computational time in the last one.

Simulations in which the $2 \mathrm{G}$ tape was only modelled by the $1 \mu \mathrm{m}$ HTS layer were compared with others where the most important layers were included, such as the substrate, the silver and the copper covering. The current distribution inside the HTS layer was the same for all the studied cases, which considered a value up to $1.03 I_{c}$. The results showed that if the transported current is not much higher than $I_{c}$, only the superconducting layer should be modelled, allowing a faster computational solution.

The model was able to calculate the external field produced by the $2 \mathrm{G}$ tape in agreement with the experimental result, presenting a maximum discrepancy about $10 \%$ and $30 \%$, for the currents rise and drop, respectively. Furthermore, fluctuations were observed along the magnetic field mapping, and they can be attributed to inhomogeneities in the sample. The maximum fluctuation observed in the $B_{y}(x, z)$ peak value of the $400 \mathrm{~A}$ and the remnant state were approximately $6 \%$ and $16 \%$, respectively. The fluctuation can partially explain the observed discrepancy between measurements and simulations, 
since if the simulation parameters were adjusted to reproduce a determined experimental profile $B_{y}(x)$ of the map, it would not fit exactly to another line.

\section{Acknowledgments}

The authors would like to thank the Brazilian Agency CNPq for the grant "Science without Borders” and the financial supported given by the European agencies EU (EU FP7-2013-ICT-609029 FORTISSIMO project, EU-FP7 NMP-LA-2012-280432 EUROTAPES project, Eurofusion PPPT-WPMAG 2014 and EU COST ACTIONS MP1201 and MP1014), MINECO (RTC-2014-1740-3) and the financial support from Spanish Ministry of Economy and Competitiveness through the "Severo Ochoa" Programme for Centres of Excellence in R\&D (SEV-2015-0496), CONSOLIDER Excellence Network (MAT2015-68994-REDC).

\section{References}

[1] Obradors X, Puig T 2014 Supercond. Sci. Technol. 27044003 doi:10.1088/09532048/27/4/044003

[2] Woodhead Publishing Series in Energy: Number 65, Superconductors in the Power Grid: Materials and Applications, Elsevier Ltd, ISBN 978-1-78242-029-3

[3] Hong Z, Campbell A, Coombs T 2006 Supercond. Sci. Technol. 19 (12) 004, pp. 1246-125, DOI: 10.1088/0953-2048/19/12/004

[4] Brambilla R, Grilli F, Martini L 2007 Supercond. Sci. Technol. 20 (1) 16-24 doi:10.1088/0953-2048/19/12/004

[5] Zermeno V, et. al. 2013 Journal of Applied Physics, 114 (17) 173901, DOI: $10.1063 / 1.4827375$

[6] Sugiura T, Hashizume H, Miya K 1991 International journal of applied electromagnetics in materials, 2 (3) 183-196.

[7] Ruiz-Alonso D, Coombs T A, Campbell A M 2004 IEEE Trans. Applied Superconductivity, 14 (4) 2053-2063. doi: 10.1109/TASC.2004.838316.

[8] Morandi A 2012 Superconductor Science and Technology, 25 (10) 104003, DOI: 10.1088/0953-2048/25/10/104003

[9] Amemiya N et. al. 1998 Phys. C, Supercond., 310 16-29

[10] Grilli F et. al.2005 IEEE Trans. Appl. Supercond., 15 (1) 17-25

[11] Stenvall A, Tarhasaari T 2010 Supercond. Sci. Technol., 23 (7) 075010

[12]Lahtinen V, Lyly M, Stenvall A and Tarhasaari T 2012 Supercond. Sci. Technol. 25 115001

[13] Francesco G et al 2014, IEEE Trans. Applied Superconductivity, (1) 8200433, DOI: 10.1109/TASC.2013.2259827

[14]Liu H et al 2015, IEEE Trans. Applied Superconductivity, 25 (3) 9000205, DOI: 10.1109/TASC.2014.2365109

[15]Pardo E, Šouc J and Kováč J 2012 Supercond. Sci. Technol. 25035003

[16]Nguyen D et al 2010 Supercond. Sci. Technol. 23025001 doi:10.1088/09532048/23/2/025001 
[17]Sass F et al 2015, Supercond. Sci. Technol., 28125012 doi:10.1088/09532048/28/12/125012

[18] Kajikawa K et al 2003, IEEE Trans. Applied Superconductivity, 13 (2) 3630, DOI: 10.1109/TASC.2003.812415

[19]Carrera M et al 2011, IEEE Trans. Applied Superconductivity, 21 (3) 3408 doi: 10.1109/TASC.2010.2089596

[20]Amoros J, Carrera M and Granados X 2012 Supercond. Sci. Technol., 25104005 doi:10.1088/0953-2048/25/10/104005

[21] Jin J 2002 The Finite Element Method in Electromagnetics (New York: Wiley)

[22] Ainslie M et al. 2011 COMPEL 30 (2) 762 DOI 10.1108/03321641111101195

[23] Navau C, Del-Valle N, and Sanchez A 2013 IEEE Trans. Applied Superconductivity, 23 (1) 8201023 DOI: 10.1109/TASC.2012.2232916

[24]Kim Y, Hempstead C, and Strnad A 1963 Phys. Rev. 129 (2) 528 DOI: 10.1103/PhysRev.129.528

[25] Irie F, and Yamafuji K 1967 J. Phys. Soc. Jpn., 23 (2) 255

[26] Green I, and Hlawiczka P 1967, Proc. IEE, 114 (9), 1329

[27] Watson J 1968 J. Appl. Phys., 39 (7) 3406

[28] Fietz W et al. 1964 Phys. Rev, 136 (2A) 335

[29] Gömöry F et al. 2009 Supercond. Sci. Technol., 22034017 DOI: 10.1088/09532048/22/3/034017

[30]Zermeño V and Grilli F 2014 Supercond. Sci. Technol., 27044025 DOI: 10.1088/0953-2048/27/4/044025

[31]Pardo E et al. 2011 Supercond. Sci. Technol., 24065007 DOI: 10.1088/09532048/24/6/065007

[32] Pardo E. and Grilli F 2012 Supercond. Sci. Technol., 25014008

[33]Molodyk K 2014 HTS Development and Industrialization at SuperOx, $1^{\text {st }}$ Workshop on Accelerator Magnets in HTS, 21-23 may, available on line at: https://indico.cern.ch/event/308828/session/2/contribution/11/attachments/589809/ 811812/2014_05_21_SuperOx_WAM-HTS.pdf

[34] Haynes W CRC Handbook of Chemistry and Physics 95 ${ }^{\text {th }}$ Ed. (CRC Press, 2015) ISBN 978-1482208672

[35]Lu J, Choi E, and Zhou H, 2008 J. Appl. Phys. 103064908

[36] Granados X, et al. Advances in Science and Technology, 47 (2006), pp. 1-6 DOI: 10.4028/www.scientific.net/AST.47.1.

[37] Carrera M et al. IEEE Trans. on App. Supercond., 21 (3), pp. 3408-3412, (2011)

DOI: 10.1109/TASC.2010.2089596 